\title{
Transcranial Doppler for Monitoring and Evaluation of Idiopathic Intracranial Hypertension
}

\author{
Pradeep R ${ }^{1} \quad$ Dhananjay Gupta ${ }^{1} \quad$ Nikith Shetty ${ }^{2} \quad$ Anjani Kumar Bhushan ${ }^{1} \quad$ Krishna Haskar $^{1}$ Sujana Gogineni ${ }^{1}$ \\ Anish Mehta ${ }^{1}$ Mahendra Javali ${ }^{1}$ Purshottam T. Acharya ${ }^{1}$ Rangasetty Srinivasa ${ }^{1}$
}

\author{
${ }^{1}$ Department of Neurology, Ramaiah Medical College, Bengaluru, \\ Karnataka, India \\ 2Department of Neurology, Kasturba Medical College, Manipal, \\ Karnataka, India
}

\begin{abstract}
Address for correspondence Dhananjay Gupta, MD, Department of Neurology, Ramaiah Medical College and Hospitals, New BEL Road, M S Ramaiah Nagar, MSRIT Post,Bengaluru 560054, Karnataka, India (e-mail: dhananjay_gupta1990@yahoo.com).
\end{abstract}

J Neurosci Rural Pract 2020;11:309-314

\begin{abstract}
Keywords

- transcranial Doppler

- ultrasonography

- cerebrospinal fluid

- CSF pressure

- headache

- papilledema

Background Idiopathic intracranial hypertension $(\mathrm{IIH})$ is a disorder of unknown origin, characterized by features of raised intracranial pressure (ICP). Existing literature is inconclusive about the role of transcranial Doppler (TCD) in the management of IIH. Objective To study the TCD changes in IIH patients, pre- and post-cerebrospinal fluid (CSF) drainage.

Materials and Methods This was a prospective study, conducted between July 2017 and December 2019, in a tertiary care referral center in South India. Sixteen consecutive patients, suspected to have $\mathrm{IH}$, underwent magnetic resonance imaging ofthe brain, a baseline TCD, and lumbar puncture with CSF drainage and pressure monitoring. Post-CSF drainage, TCD was repeated and mean flow velocities, peak systolic velocities, end-diastolic velocities, and pulsatility index (PI), in the middle cerebral artery (MCA), vertebral artery, and basilar artery (BA) were noted. Thirteen patients had elevated CSF pressure, and fulfilled the diagnostic criteria for IIH. These patients were included in the final analysis and pre- and post-CSF drainage TCD blood flow velocities and PI were compared.

Results The mean age of study participants was $29.92 \pm 6.92$ years. There was a significant reduction in the cerebral flow velocities in bilateral MCA, after CSF drainage and normalization of ICP. Flow velocities in posterior circulation and PI in MCA, PCA, and BA showed an insignificant reduction. Two patients, who did not show any reduction in flow velocities after CSF drainage, developed optic atrophy on follow-up.

Conclusion TCD-derived systolic blood flow velocities can be used in the management and follow-up of patients with $\mathrm{IH}$.
\end{abstract}

\section{Introduction}

Idiopathic intracranial hypertension (IIH) is a disorder of unknown cause, presenting with signs and symptoms of raised intracranial pressure (ICP), in the absence of underlying structural or other identifiable causes. The clinical course is not always benign and, left untreated, can lead to permanent loss of vision. ${ }^{1}$ In population-based studies, the annual incidence of IIH has been reported to be 0.9 per 100,000 population. ${ }^{2}$ Females are eight times more commonly affected as compared with males and incidence increases manyfolds in overweight and obese patients. ${ }^{3}$ With the recent global obesity epidemic, the incidence of $\mathrm{IIH}$ is on a rise and it is becoming an area of interest for researchers. Diagnosis of IIH by the modified Dandy criteria involves invasive measurement of ICP. ${ }^{4}$ ICP measurement
DOI https://doi.org/ $10.1055 / \mathrm{s}-0040-1710086$ ISSN 0976-3147.
C2020 Association for Helping Neurosurgical Sick People
License terms

$($ (1) $\Theta \circledast$ 
for monitoring response to therapy may require repeated lumbar punctures, and is uncomfortable for the patient as well as the physician. To overcome this, noninvasive methods, such as transcranial Doppler (TCD) and ultrasonography of the optic nerve sheath diameter (ONSD) are being evaluated as indirect measures of ICP.

In recent studies, TCD has shown promise in experimental models of intracranial hypertension and circulatory arrest. ${ }^{5}$ A significant correlation between ICP and the TCD-derived pulsatility index (PI) values has been shown in neurocritical patients, including those with head trauma, subarachnoid hemorrhage, and acute bacterial and tuberculous meningitis. ${ }^{6-12}$ Studies in patients with IIH are limited and have reported contrasting results. Gur et al showed no unique TCD features for monitoring IIH patients. ${ }^{13}$ On the contrary, Hunter et al showed that TCD-derived PI values changed significantly with change in ICP and suggested potential role of TCD in noninvasive monitoring of IIH. ${ }^{14}$ Hence, we evaluated the changes in TCD parameters, before and after therapeutic cerebrospinal fluid (CSF) drainage, in patients diagnosed with IIH.

\section{Materials and Methods}

This was a prospective trial undertaken in a tertiary referral center in southern India. An institutional ethical clearance was taken before starting the study. Patients were recruited between July 2017 and December 2019. Sixteen consecutive patients, suspected to have IIH, based on clinical features (headache, vomiting, visual disturbances, abducens palsy, and papilledema) were enrolled for the study, after taking an informed consent ( $\boldsymbol{- F i g . 1}$ ). All these patients underwent brain imaging (computed tomography or magnetic resonance imagingalong with venography of brain) to exclude an underlying structural cause, including cerebral venous sinus thrombosis. If the neuroimaging was normal or the findings were consistent with IIH, patients underwent a baseline TDC

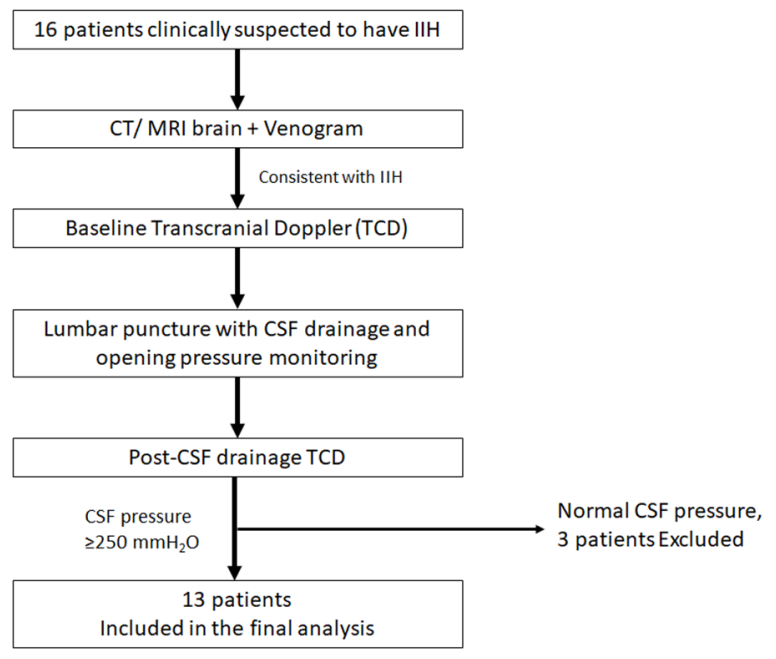

Fig. 1 Study design.CSF, cerebrospinal fluid; CT, computed tomography; $\mathrm{IH}$, idiopathic intracranial hypertension; MRI, magnetic resonance imaging. study (Nicolet Sonara TCD system, Natus neurology, United States) to estimate the mean flow velocity (MFV), peak systolic velocity (PSV), end-diastolic velocity (EDV), PI, and resistance index (RI) in the intracranial vessels. TCD was performed by investigator no. 1 , with the patient in supine position and both the anterior (middle cerebral artery[MCA]) and the posterior circulation (vertebral artery[VA] and basilar artery[BA]) were studied. The MCA was studied through the transtemporal window with insonation depth of 50 to $55 \mathrm{~mm}$. The VA and BA were studied through the suboccipital window and transforaminal window. Following this, patients underwent lumbar puncture with CSF opening pressure monitoring. Lumbar puncture was done in the left lateral decubitus position (performed by investigator no. 2 , who was blinded to the TCD results). Thirteen out of 16 patients had elevated CSF pressures, $>250 \mathrm{mmH}_{2} \mathrm{O}$, and in these, $\sim 30 \mathrm{~mL}$ of CSF was drained to achieve a normal closing pressure. CSF samples were analyzed for routine cell count, cell types, and sugar and protein levels. All the patients underwent postCSF drainage TCD, within 60 minutes of the lumbar puncture. Investigator no. 1 performed the post-CSF drainage TCD and was blinded to the lumbar puncture results. Thirteen patients, who had elevated CSF pressures with a normal CSF biochemical testing, were included for the final analysis.

\section{Statistical Analysis}

All analyses were done using the IBM-SPSS software (version 20.0). Data are presented as mean \pm standard deviation. The normality of data was tested using Kolmogorov-Smirnov's test and data were found to be following normal distribution. Pre- and post-CSF drainage TCD velocities and PI values were compared using the paired $t$-test. Pearson's coefficient was used to correlate MFV, PSV, EDV, and PI values with the CSF pressure. Linear regression analysis was used to evaluate effects of variables such as weight and body mass index (BMI) on the results of CSF pressure and TCD-derived flow velocities. All values were considered significant if $p$-value was $<0.05$.

\section{Results}

Thirteen of the 16 eligible participants fulfilled the diagnostic criteria of IIH and were included in the final analysis. Ten females and three males, with mean age of $29.92 \pm 6.92$ years were studied over a period of 2 years ( $\boldsymbol{- T a b l e ~} \mathbf{1}$ ). All these patients had either normal brain imaging or features consistent with a diagnosis of IIH. Mean CSF opening pressure in study population was $307.7 \pm 46.8 \mathrm{~mm} \mathrm{H}_{2} \mathrm{O}$, with a normal CSF biochemical analysis. Pre- and post-CSF drainage TCD values are shown in - Table 2 .

1. Pre-and post-PSVs: The PSVs in both the right and left MCAs reduced significantly following CSF drainage $(p=0.001$ and 0.003 , respectively). In the posterior circulation, PSV was reduced post-CSF drainage, but did not reach levels of statistical significance in the BA or the left VA.

2. Pre- and post-MFVs: Similar to PSV, the systolic MFVs in bilateral MCA reduced significantly post-CSF drainage ( $p=0.001$ and 0.003 , respectively). There was nonsignificant reduction in MFVs in both the VA and BA. 
Table 1 Demographic data and CSF analysis in study population

\begin{tabular}{|c|c|c|c|}
\hline$N=13$ & Minimum & Maximum & Mean \pm SD \\
\hline Age (y) & 16 & 40 & $29.92 \pm 6.92$ \\
\hline Height (cm) & 154 & 170 & $162.1 \pm 5.88$ \\
\hline Weight (kg) & 62 & 119 & $76.15 \pm 15.46$ \\
\hline $\mathrm{BMI}\left(\mathrm{kg} / \mathrm{m}^{2}\right)$ & 24 & 49 & $30.15 \pm 5.14$ \\
\hline CSF opening pressure $\left(\mathrm{cmH}_{2} \mathrm{O}\right)$ & 25 & 40 & $30.77 \pm 4.68$ \\
\hline CSF cells & 0 & 4 & $2 \pm 1.29$ \\
\hline CSF protein (mg/dL) & 13 & 59 & $33.46 \pm 15.13$ \\
\hline CSF glucose (mg/dL) & 43 & 78 & $63.77 \pm 11.41$ \\
\hline
\end{tabular}

Abbreviations: BMI, body mass index; CSF, cerebrospinal fluid; SD, standard deviation.

Table 2 Transcranial Doppler findings in study population

\begin{tabular}{|c|c|c|c|}
\hline & Pre-CSF drainage & Post-CSF drainage & $p$-Value \\
\hline \multicolumn{4}{|c|}{ Right middle cerebral artery } \\
\hline PSV & $97.21 \pm 16.69$ & $80.52 \pm 16.22$ & 0.001 \\
\hline MFV & $59.78 \pm 11.91$ & $50.33 \pm 11.02$ & 0.001 \\
\hline EDV & $39.62 \pm 8.07$ & $34.63 \pm 11.10$ & 0.013 \\
\hline $\mathrm{PI}$ & $0.95 \pm 0.12$ & $0.90 \pm 0.17$ & 0.213 \\
\hline \multicolumn{4}{|c|}{ Left middle cerebral artery } \\
\hline PSV & $93.96 \pm 22.01$ & $81.15 \pm 18.90$ & 0.003 \\
\hline MFV & $59.89 \pm 18.60$ & $52.62 \pm 15.51$ & 0.003 \\
\hline EDV & $41.25 \pm 14.00$ & $37.35 \pm 11.91$ & 0.062 \\
\hline $\mathrm{Pl}$ & $0.89 \pm 0.19$ & $0.85 \pm 0.16$ & 0.552 \\
\hline \multicolumn{4}{|c|}{ Basilar artery } \\
\hline PSV & $62.64 \pm 8.70$ & $59.78 \pm 10.16$ & 0.249 \\
\hline MFV & $40.89 \pm 7.63$ & $40.03 \pm 7.93$ & 0.529 \\
\hline EDV & $27.33 \pm 6.90$ & $26.42 \pm 5.64$ & 0.756 \\
\hline $\mathrm{PI}$ & $0.86 \pm 0.15$ & $0.82 \pm 0.12$ & 0.463 \\
\hline \multicolumn{4}{|c|}{ Right vertebral artery } \\
\hline PSV & $48.18 \pm 10.15$ & $44.51 \pm 7.35$ & 0.006 \\
\hline MFV & $30.11 \pm 5.82$ & $30.11 \pm 5.82$ & 0.197 \\
\hline EDV & $20.79 \pm 4.25$ & $19.41 \pm 3.37$ & 0.398 \\
\hline $\mathrm{PI}$ & $0.85 \pm 0.21$ & $0.80 \pm 0.16$ & 0.328 \\
\hline \multicolumn{4}{|c|}{ Left vertebral artery } \\
\hline PSV & $51.15 \pm 9.91$ & $49.33 \pm 10.99$ & 0.388 \\
\hline MFV & $32.25 \pm 7.13$ & $31.99 \pm 6.85$ & 0.861 \\
\hline EDV & $21.42 \pm 5.45$ & $21.81 \pm 4.84$ & 0.656 \\
\hline d. PI & $0.92 \pm 0.21$ & $0.85 \pm 0.16$ & 0.363 \\
\hline
\end{tabular}

Abbreviations: CSF, cerebrospinal fluid; EDV, end-diastolic velocity; MFV, mean flow velocity; PI, pulsatility index; PSV, peak systolic velocity.

3. Pre- and post-PIs: Although PI values in all the studied intracranial arteries decreased concurrently with decrease in CSF pressure, none of them reached levels of statistical significance.

4. Correlation of PSV and MFV with CSF opening pressure (-Fig. 2): Baseline or pre-CSF drainage blood flow velocities in MCA had a strong correlation with CSF opening pressure (Pearson's correlation coefficients are 0.927 for PSV, 0.80 for MFV, and 0.91 for EDV; $p<0.05$ ). Blood flow velocities in the BA and VAs had a weaker positive correlation (Pearson's correlation coefficient 0.12-0.63).

5. Effect of weight and BMI on CSF opening pressure and flow velocities: CSF opening pressure showed a stronger correlation with BMI (Pearson's coefficient 0.46) compared with body weight (Pearson's coefficient 0.35 ), though both were statistically nonsignificant. When subgrouped by gender, the correlation was stronger in females compared with males. On the contrary, intracranial blood flow 

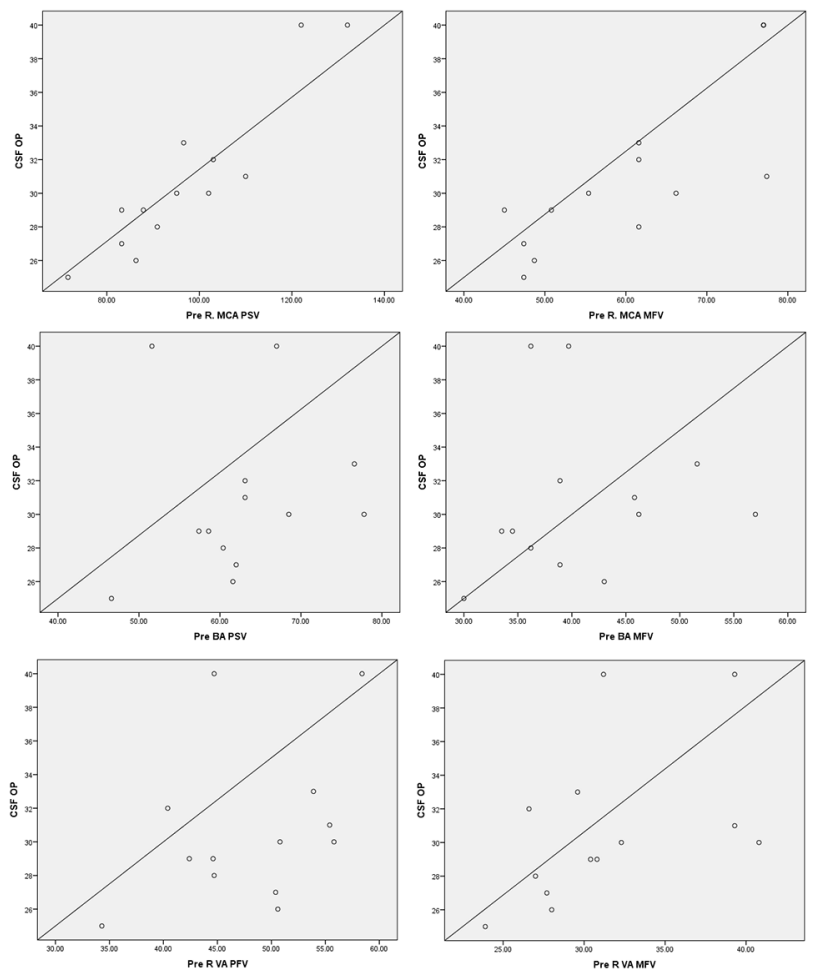

Fig. 2 Correlation of cerebral blood flow velocities with CSF-opening pressure. BA, basilar artery; EDV, end-diastolic velocity; L, left; MCA, middle cerebral artery; MFV, mean flow velocity; PI, pulsatility index; PSV, peak systolic velocity; R, right; VA, vertebral artery. velocities did not show a consistent correlation with either weight or BMI.

6. Predicting reduction in flow velocities: Further, we tried to ascertain if the amount of change or reduction in systolic and diastolic velocities could be predicted by baseline patient characteristics such as gender, height, weight, BMI, or CSF opening pressure. However, none of these variables showed a consistent correlation with reduction in flow velocities and was not found to be statistically significant.

7. Follow-up: Two of the 13 patients with IIH did not show any significant decrease in PSV or MFV postCSF drainage, despite normalization of CSF pressures. Even with appropriate medical management, these patients remained symptomatic. Both were found to have developed chronic papilledema/optic atrophy, on follow-up after 2 months.

\section{Discussion}

This is the first of its kind of study in a South Asian cohort. Previously, we could only find two similar studies, one done in Canada and other in Israel, both showing conflicting results..$^{13,14}$ Our study participants were between 16 and 40 years of age and could be classified as overweight or obese, as per the World Health Organization criteria (BMI $\left.>25 \mathrm{~kg} / \mathrm{m}^{2}\right) .{ }^{15}$ The initial peak and mean blood flow velocities (PSV and MFV) in most of our study population were within the upper limits of normal. This is similar to Gur et al, who found that blood velocities, PI, and RI of IIH patients were higher than normal controls, but still within the upper normal limit. ${ }^{13}$ It has been suggested that cerebral regulatory functions are still effective during
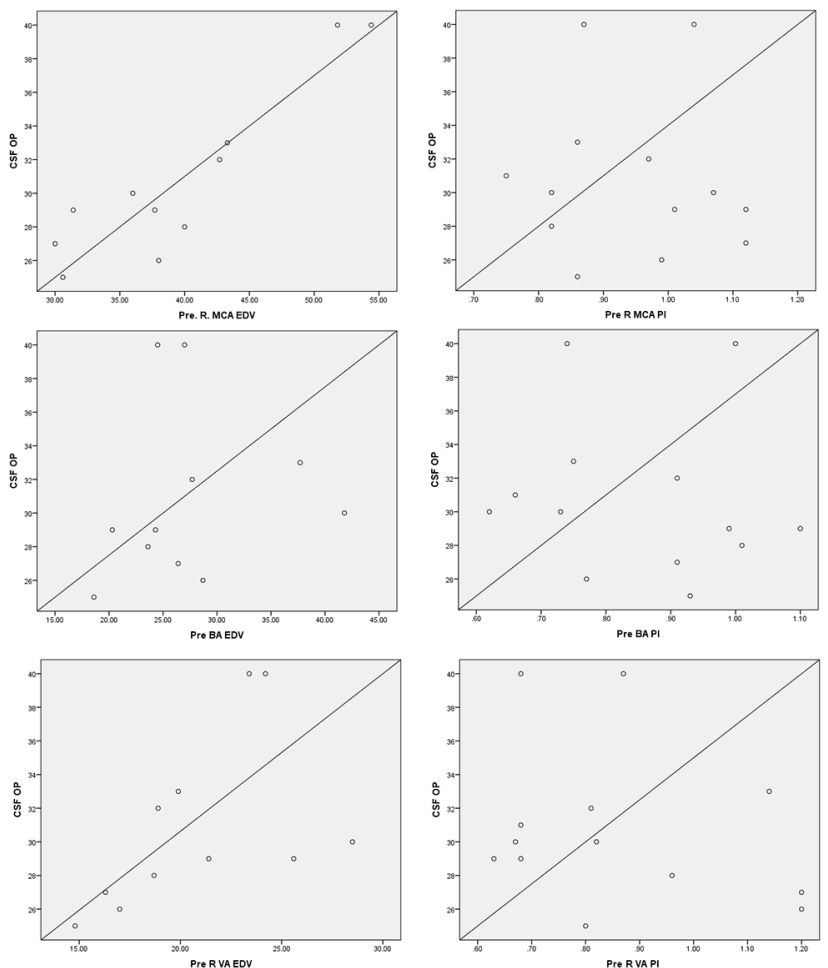

elevations of ICP as seen in IIH. Hence, the systolic blood flow velocities do not increase as much as expected in patients with acute severe elevations of ICP, as in cases of traumatic brain injury or subarachnoid hemorrhage. ${ }^{16,17}$ However, it has been consistently shown that peak and mean systolic as well as diastolic flow velocities are significantly higher in patients with IIH when compared with controls with normal CSF pressure. ${ }^{13,18}$

The baseline blood flow velocities in the MCA correlated significantly with the CSF opening pressure in the study population, irrespective of the gender, height, weight, or BMI of the participants (-Fig. 2). We repeated the TCD measurements immediately after CSF drainage and normalization of ICP and found a uniform reduction in systolic as well as diastolic flow velocities. The reduction was more significant in anterior circulation (MCA) as compared with posterior circulation (VA and BA). The reason for the same is yet to be elucidated. Whether the higher cerebral blood flow in anterior circulation (three times as compared with posterior circulation $)^{19}$ or the difference in autonomic innervation of anterior and posterior arteries ${ }^{20}$ plays a role needs further dedicated studies. One previous study has studied blood flow changes after CSF drainage in females with high CSF opening pressure. However, the exact vessels studies have not been mentioned by the authors. ${ }^{21}$

Conversely, some researchers have tried to correlate changes in PI and the RI with changes in ICP with contrasting results. While Hunter et $\mathrm{al}^{14}$ demonstrated a significant reduction of PI after normalization of ICP with CSF drainage, another small study in five female patients failed to do so. ${ }^{21}$ In our cohort, there was an insignificant reduction in PI values 
post-CSF drainage. It is essential to remember that PI is a complex measurement and is affected by many interdependent hemodynamic variables including cerebral perfusion pressure (CPP), cerebrovascular resistance, compliance of arterial bed, and the heart rate. ${ }^{22}$ It reflects the CPP more accurately than ICP, especially in cases of moderate ICP elevations in more chronic disorders such as IIH. Recently, newer models of IIH, focusing on the role of intracerebral veins, venous pressure and CSF changes, and the role of brain parenchyma morphological changes have been proposed. ${ }^{23}$

Two of our patients failed to show a significant decrease in TCD parameters post-CSFdrainage and normalization of CSF pressure. They were followed up closely, and were found to develop chronic papilledema. Whether nonreduction of TCD parameters post-CSF drainage can serve as a marker for adverse prognosis in such patients needs to be studied in further larger prospective studies.

\section{Limitations}

This was a pilot study with a small sample size. We did not have a matched cohort of healthy individuals to compare the TCD values of IIH patients. IIH is a chronic disease with periods of relapses and remissions, as such, more number of follow-up visits are needed to prove an unequivocal role of TCD in the management and follow-up of IIH patients. We have also not evaluated the ONSD, which is presently the method of choice for estimation of ICP.

\section{Conclusion}

TCD-derived peak and MFVs can be used as a noninvasive adjunctive tool for management, prognostication, and follow-up in patients with IIH.

\section{Significance Statement}

Due to the global obesity epidemic, the incidence of IIH is ever increasing. There is an unmet need to evaluate noninvasive diagnostic tests for IIH. Here, we have evaluated and found noninvasive modality, TCD for management of such patients.

\section{Key Findings}

- A noninvasive modality, TCD is useful in the clinical management of patients with IIH.

- Cerebral blood flow velocities reduce after normalization of ICP, post-CSF drainage.

- Failure of reduction of blood flow velocities may indicate adverse prognosis.

\section{Note}

The data that support the findings of this study are available on request from the corresponding author. The data are not publicly available due to privacy or ethical restrictions.

\section{Ethical Approval}

Institutional ethics committee approval was obtained, No 10/2019.

\section{Authors' Contributions}

P. R.: Conceptualization, investigation, performed TCD for all patients

D. G.: Conceptualization, investigation, performed lumbar puncture for all patients

N. S.: Writing-original draft

A. K. B.: Methodology

K. H.: Writing-original draft

S. G.: Writing-review andediting

A. M.: Methodology

M. J.: Formal analysis

P. T. A.: Writing-review andediting

R. S.: Supervision.

\section{Funding}

None.

\section{Conflict of Interest}

None declared.

\section{References}

1 Wakerley BR, Tan MH, Ting EY. Idiopathic intracranial hypertension. Cephalalgia 2015;35(3):248-261

2 Durcan FJ, Corbett JJ, Wall M. The incidence of pseudotumor cerebri. Population studies in Iowa and Louisiana. Arch Neurol 1988;45(8):875-877

3 Radhakrishnan K, Thacker AK, Bohlaga NH, Maloo JC, Gerryo SE. Epidemiology of idiopathic intracranial hypertension: a prospective and case-control study. J Neurol Sci 1993;116(1):18-28

4 Friedman DI, Jacobson DM. Diagnostic criteria for idiopathic intracranial hypertension. Neurology 2002;59(10):1492-1495

5 Nagai H, Moritake K, Takaya M. Correlation between transcranial Doppler ultrasonography and regional cerebral blood flow in experimental intracranial hypertension. Stroke 1997;28(3):603-607, discussion 608

6 Czosnyka M, Matta BF, Smielewski P, Kirkpatrick PJ, Pickard JD. Cerebral perfusion pressure in head-injured patients: a noninvasive assessment using transcranial Doppler ultrasonography. J Neurosurg 1998;88(5):802-808

7 Bellner J, Romner B, Reinstrup P, Kristiansson K-A, Ryding E, Brandt L. Transcranial Doppler sonography pulsatility index (PI) reflects intracranial pressure (ICP) Surg Neurol 2004;62(1):45-51, discussion 51

8 Voulgaris SG, Partheni M, Kaliora H, Haftouras N, Pessach IS, Polyzoidis KS. Early cerebral monitoring using the transcranial Doppler pulsatility index in patients with severe brain trauma. Med Sci Monit 2005;11(2):CR49-CR52

9 Tai MS, Sharma VK. Role of transcranial Doppler in the evaluation of vasculopathy in tuberculous meningitis. PLoS One 2016;11(10):e0164266

10 van Toorn R, Schaaf HS, Solomons R, Laubscher JA, Schoeman JF. The value of transcranial Doppler imaging in children with tuberculous meningitis. Childs NervSyst 2014;30(10):1711-1716

11 Lu CH, Chang HW, Lui CC, Huang CR, Chang WN. Cerebral haemodynamics in acute bacterial meningitis in adults. QJM 2006;99(12):863-869

12 Kumari R, Toppo SK, Ram PN, Ranjan RK, Deivigan P, Agarwal V. Utility of serial transcranial Doppler in diagnosis of intracranial vasculopathy with computed tomography correlation in children with tuberculous meningitis. J MedSciClin Res 2019;7(1)

13 Gur AY, Kesler A, Shopin L, Bornstein NM. Transcranial Doppler for evaluation of idiopathic intracranial hypertension. Acta NeurolScand 2007;116(4):239-242 
14 Hunter G, Voll C, Rajput M. Utility of transcranial doppler in idiopathic intracranial hypertension. Can J NeurolSci 2010;37(2):235-239

15 World Health Organization. Obesity. Preventing and managing the global epidemic. Report on a WHO Consultation on Obesity. Technical Report Series Number 894. Geneva. World Health Organization; 2000

16 Giulioni M, Ursino M, Alvisi C. Correlations among intracranial pulsatility, intracranial hemodynamics, and transcranial Doppler wave form: literature review and hypothesis for future studies. Neurosurgery 1988;22(5):807-812

17 Djelilovic-Vranic J, Basic-Kes V, Tiric-Campara M, Djozic E, Kulenovic J. Follow-up of vasospasm by transcranial Doppler sonography (TCD) in subarachnoid hemorrhage (SAH) Acta Inform Med 2017;25(1):14-18

18 Bateman GA. Vascular hydraulics associated with idiopathic and secondary intracranial hypertension. AJNR Am J Neuroradiol 2002;23(7):1180-1186
19 Zarrinkoob L, Ambarki K, Wåhlin A, Birgander R, Eklund A, Malm J. Blood flow distribution in cerebral arteries. J Cereb Blood Flow Metab 2015;35(4):648-654

20 Roloff EV, Tomiak-Baquero AM, Kasparov S, Paton JF. Parasympathetic innervation of vertebrobasilar arteries: is this a potential clinical target? J Physiol 2016;594(22):6463-6485

21 Martinez-Prieto JN. Decreased pulsatility index measured by transcranial Doppler (TCD) in patients with idiopathic intracranial hypertension (pseudotumor cerebri) Cerebrovasc Dis 1999;9(Suppl 2):10

22 de Riva N, Budohoski KP, Smielewski P, et al. Transcranial Doppler pulsatility index: what it is and what it isn't. Neurocrit Care 2012;17(1):58-66

23 Mangalore S, Rakshith S, Srinivasa R. Solving the riddle of "Idiopathic" in idiopathic intracranial hypertension and normal pressure hydrocephalus: An imaging study of the possible mechanisms - Monro-Kellie 3.0. Asian J Neurosurg 2019;14(2):440-452 